University of Wollongong

Research Online

Faculty of Engineering and Information

Faculty of Engineering and Information

Sciences - Papers: Part B

Sciences

2020

\title{
Robustness in coupling between iron and rare earth spins in rare earth orthoferrites
}

Mohanad Hazim

University of Wollongong

Josip Horvat

University of Wollongong, jhorvat@uow.edu.au

Zhenxiang Cheng

University of Wollongong, cheng@uow.edu.au

Shixun Cao

Follow this and additional works at: https://ro.uow.edu.au/eispapers1

Part of the Engineering Commons, and the Science and Technology Studies Commons

Research Online is the open access institutional repository for the University of Wollongong. For further information contact the UOW Library: research-pubs@uow.edu.au 


\title{
Robustness in coupling between iron and rare earth spins in rare earth orthoferrites
}

\author{
Abstract \\ (C) 2020 Trans Tech Publications Ltd, Switzerland. We report on very accurate magnetic measurements on \\ large rare earth orthoferrites single crystals of $\mathrm{ErFeO} 3$ and $\mathrm{NdFeO3}$. Our results show that the interaction \\ between rare earth and iron spin system does not change during the spin-flip process. This implies that \\ the coupling between the iron and rare earth spin systems is robust enough to withstand the effects of \\ spin flipping against the magnetic anisotropy energy. This is despite rare eath ions, polarized by the \\ ordered iron ions, being in partly metastable state and their magnetic moment decays with time.

\section{Disciplines} \\ Engineering | Science and Technology Studies

\section{Publication Details} \\ Hazim, M., Horvat, J., Cheng, Z. \& Cao, S. (2020). Robustness in coupling between iron and rare earth \\ spins in rare earth orthoferrites. Materials Science Forum, 985 MSF 29-34.
}




\title{
Robustness in coupling between iron and rare earth spins in rare earth orthoferrites
}

\author{
Mohanad Hazim Mohammed ${ }^{1, a^{*}}$ Joseph Horvat $^{1, \mathrm{~b}}$, Zhenxiang Cheng ${ }^{1,2, \mathrm{c}}$, Shixun \\ $\mathrm{CaO}^{2, \mathrm{~d}}$ \\ ${ }^{1}$ Institute for Superconducting and Electronic Materials and School of Physics, University of \\ Wollongong, NSW 2522, Australia \\ ${ }^{2}$ Department of Physics, Shanghai University, 99 Shangda Road, Shanghai 200444, China \\ a*mhm670@uowmail.edu.au, b jhorvat@uow.edu.au, c cheng@uow.edu.au \\ dsxcao@shu.edu.cn
}

Keywords: Rare earth orthoferrites, Spintronic materials

\begin{abstract}
We report on very accurate magnetic measurements on large rare earth orthoferrites single crystals of $\mathrm{ErFeO}_{3}$ and $\mathrm{NdFeO}_{3}$. Our results show that the interaction between rare earth and iron spin system does not change during the spin-flip process. This implies that the coupling between the iron and rare earth spin systems is robust enough to withstand the effects of spin flipping against the magnetic anisotropy energy. This is despite rare eath ions, polarized by the ordered iron ions, being in partly metastable state and their magnetic moment decays with time.
\end{abstract}

\section{Introduction}

Since 1950's when Forestier and Guit-Guillaind first time found the rare earth orthoferrites materials $R \mathrm{MO}_{3}(R$ and $\mathrm{M}$ represents a trivalent rare-earth ion and transition metals $(\mathrm{Fe}, \mathrm{Co}$, $\mathrm{Ni}$ ), respectively), these materials attracted attention from many researchers due to their remarkable magnetic and optical features [1-4]. The magnetic properties and phase transition of rare earth orthoferrites $R \mathrm{FeO}_{3}$ such as spin reorientation (SR), canted antiferromagnetism ( $A F M)$, net ferromagnetism $(F M)$ and ordering of the $R^{3+}$ moment at low temperature have been studied considerably[5-12]. The magnetic moment for single crystal samples $\mathrm{ErFeO}_{3}$ and $\mathrm{NdFeO}_{3}$ have been studied before [13,14]. Although $\mathrm{Nd}^{3+}$ and $\mathrm{Er}^{3+}$ ions order antiferromagnetically only at low temperatures $\left(T_{\mathrm{N}}^{\mathrm{Nd}}=1.25 \mathrm{~K}, T_{\mathrm{N}}^{\mathrm{Er}}=4.4 \mathrm{~K}\right)$ [15], they still have a key role in magnetic properties in the whole system above these temperatures. This is because the net magnetic moment of the ordered $\mathrm{Fe}^{+3}$ ions can polarize $R^{3+}$ ions at temperature $T>T_{\mathrm{N}}^{\mathrm{R}}$ [5,9], giving them a non-zero net magnetic moment. There is relatively strong coupling between the partially polarized $R^{3+}$ and $\mathrm{Fe}^{3+}$ spins. Therefore, the interaction between $R^{3+}$ and $\mathrm{Fe}^{3+}$ ions 
at different temperatures can play a key role in magnetic transitions for $R \mathrm{FeO}_{3}[16]$. Reports show there is an abrupt jump of the overall magnetic moment with cooling, spin-flip, which is in addition to more frequently studied gradual spin reorientation process at higher temperatures $[1-3,13,14]$. Further work on this phenomenon was suggested, to understand the principles and reasons for that jump. Study of these materials is of interest to the development of spintronic materials, where the electron spin is utilized instead of its charge as a means of information encoding.

In this work, we study the magnetic moment of $\mathrm{ErFeO}_{3}$ and $\mathrm{NdFeO}_{3}$ single crystal samples in the vicinity of the spin-flip temperature. We focused on the irreversibility of the spin-flip process. The accurate measurements of $M(T)$ could help to understand the characteristics of the magnetic exchange interaction between iron and rare-earth spins. We confirmed there is strong coupling between $\mathrm{Fe}^{3+}$ and $R^{3+}$ which is not perturbed by a whole system rotation in the spin-flip region.

\section{Experimental work:}

The measurements were performed on two single crystals, $\mathrm{ErFeO}_{3}$ and $\mathrm{NdFeO}_{3}$ with the weights $0.264 \mathrm{~g}$ and $0.3733 \mathrm{~g}$, respectively. The temperature range of measurements was $300-$ $5 \mathrm{~K}$ with different applied magnetic fields. Our single crystals were grown using the fourmirror optical floating-zone furnace (FZ-T-10000-H-VI-P-SH, Crystal System Corp.) by Prof. Shixun Cao's group at Shanghai University, China[17,18]. A mixture of $99.99 \%$ pure powders of $\mathrm{R}_{2} \mathrm{O}_{3}$ and $\mathrm{Fe}_{2} \mathrm{O}_{3}(99.99 \%)$ was used in the stoichiometric ratio. The powders were initially sintered at $1100{ }^{\circ} \mathrm{C}$, then crushed and ground. Further sintering at $1350{ }^{\circ} \mathrm{C}$ resulted in polycrystalline seed-rods. These rods were used for single crystal growth in a floating zone furnace. The furnace had four ellipsoidal mirrors, with four $1.0 \mathrm{~kW}$ halogen lamps as the heat sources. The rotation of the feed and seed shafts was in the opposite direction. The crystals were grown in flowing air in the vertical direction, from bottom to the top. The melting zone travelling rate was in the range of $2.5-7.5 \mathrm{~mm} / \mathrm{h}$. The single crystal samples in this study were examined by X-ray Laue photography, to ascertain the crystallinity and crystallographic orientations. The back-reflections of Laue XRD patterns confirmed the orthorhombically distorted perovskite structure with Pbnm symmetry for the single crystal samples

The magnetic measurements were implemented using the vibrating sample magnetometer (VSM) option of a Physical Property Measurement System (PPMS-9, Quantum Design). The frequency of VSM vibration was $40 \mathrm{~Hz}$ and amplitude $2 \mathrm{~mm}$. The measurements of the 
temperature dependence of magnetic moment, $M(T)$, were conducted with the field-cooledcooling procedure (FCC).

\section{Results and discussion}

Figure 1. shows the hysteresis loops for $\mathrm{ErFeO}_{3}$ and the $\mathrm{NdFeO}_{3}$ samples, measured along the $a$ - and $c$-axes. The measurements were performed in different ranges of temperatures through the spin reorientation temperature range, SRT.
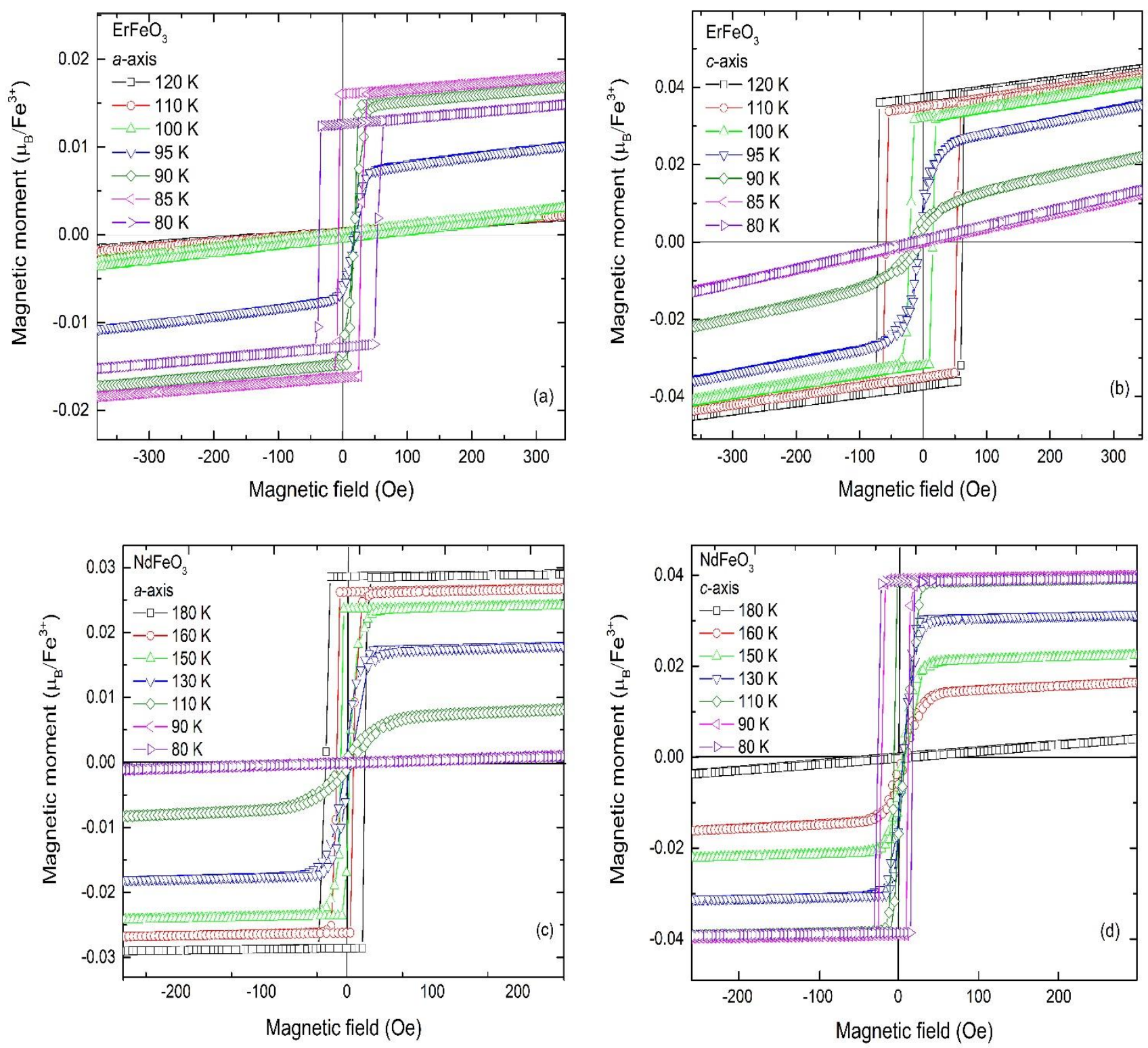

Figure 1. Magnetic hysteresis loops. (1) $\mathrm{ErFeO}_{3}$ : (a) measured along the crystalline $a$-axis, (b) measured along the crystalline $c$-axis. (2) $\mathrm{NdFeO}_{3}$ : (c) measured along the crystalline $a-$ axis, (d) measured along the crystalline $c$-axis. 
The data indicates that the magnetic behaviour of the two samples is similar. The following conclusions can be drawn: (a) Above the SRT for $\mathrm{ErFeO}_{3}$ and $\mathrm{NdFeO}_{3}$, the magnetic moment along the $a$-axis, $M a$, displays reversible straight lines going through the origin with equivalent temperature-independent slopes, while it has a rectangular shape when hysteresis loops are measured along the $c$-axis for both samples; (b) Below the SRT, the shape of the hysteresis loops is rectangular along the $a$-axis, but in the form of straight lines along the $c$-axis; (c) in the SRT region, the magnetization curves are S-shaped. These measurements of hysteresis loops are consistent with the conclusions of previous reports and can be described with theoretical models $[14,16,19-21]$. The domain wall jumps are shown in the $M(H)$ curves by abrupt changes of magnetization, which represent extrinsic properties of the crystals [14,21]. Their effects on $M(T)$ can be avoided by measuring at high enough fields, at which $M(H)$ is reversible, and there are no jumps in the hysteresis loops. Because of that, all $M(T)$ measurements were performed in the high-field, reversible regime, to avoid extrinsic effects on the measurements (i.e. effects of crystal defects). Typically, applied field $H>100$ Oe was needed in $M(T)$ measurements to avoid domain wall jumps at all temperatures.

In $\mathrm{RFeO}_{3}$ studied here, $\mathrm{Fe}^{3+}$ spins are ordered antiferromagnetically, with an additional tilt that gives a net ferromagnetic moment along crystalline $c$-axis at room temperature. Rare earth ions
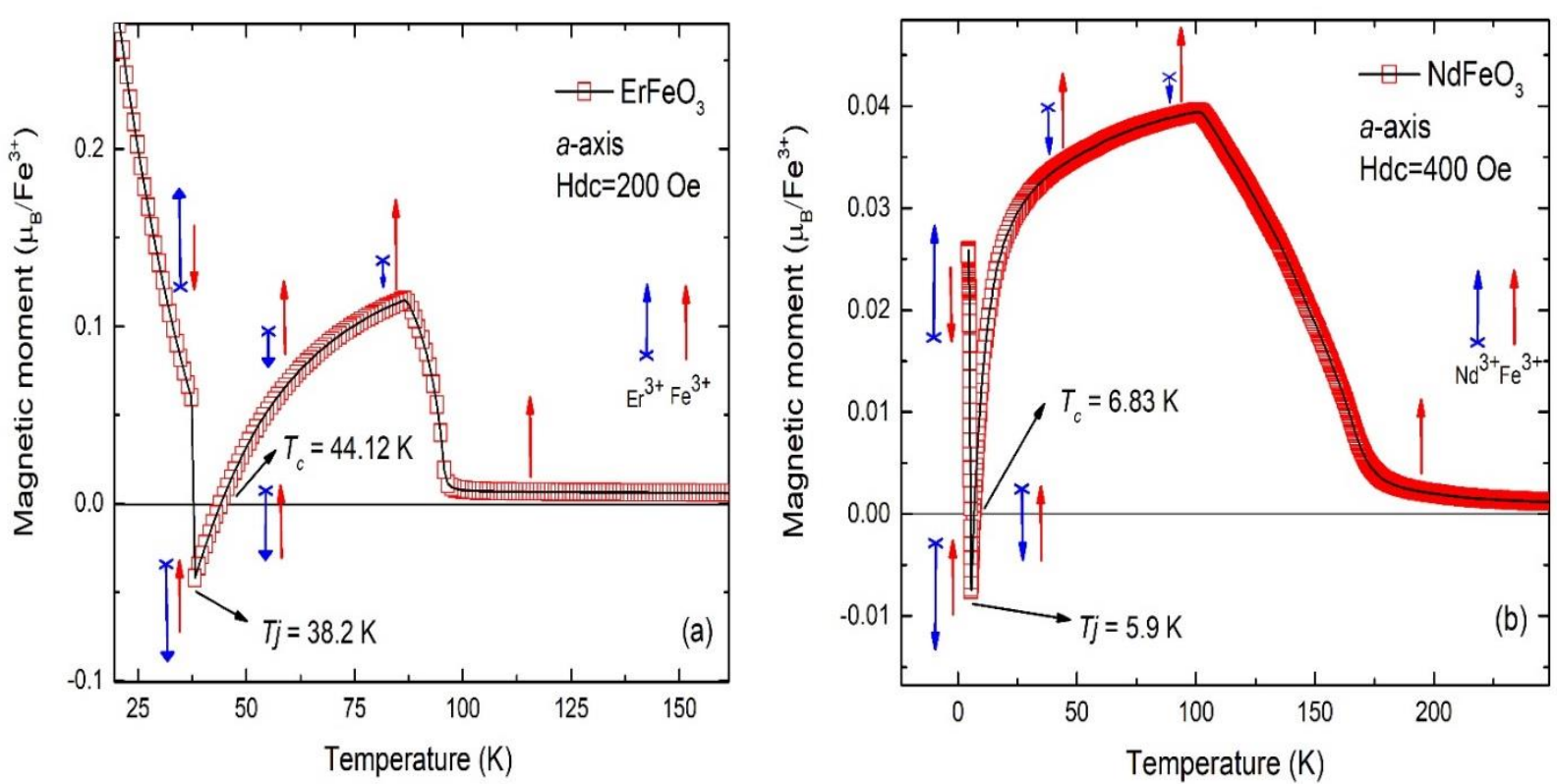

Figure 2. Temperature dependence of magnetic moment measured along crystalline $a$-axis:

(a) $M-T$ curve for $\mathrm{ErFeO}_{3}$ single crystal, (b) $M-T$ curve for $\mathrm{NdFeO}_{3}$ single crystal. 
are not ordered but are polarized by the ordered iron ions. Polarized $R^{3+}$ ions have in average a small net magnetic moment $(M)$ in the direction opposite to the net magnetic moment of $\mathrm{Fe}^{3+}$.

Decreasing the temperature $(T)$, the whole magnetic system undergoes a spontaneous spin reorientation of the net magnetic moment from $c$ - to $a$-axis in the temperature range of 88-100 $\mathrm{K}$ for $\mathrm{ErFeO}_{3}$ and 104-167 $\mathrm{K}$ for $\mathrm{NdFeO}_{3}$.

As the rare earth ions are polarized in the opposite direction to the net moment of $\mathrm{Fe}^{3+}$ ions, the overall sample moment along $a$-axis starts to decrease below the spin reorientation transition. With further cooling in the magnetic field along the $a$-axis for both samples, Fig.2 (a) and (b), the polarization of the rare earth spin becomes stronger, while the net moment of $\mathrm{Fe}^{3+}$ does not change much. When the magnetic moment of $R^{3+}$ and $\mathrm{Fe}^{3+}$ are equal in the absolute values and opposite in the directions, the total magnetization will be zero. That happens at temperature named the compensation temperature $T_{c}$. For the fields shown in Fig. 2, the field-dependent $T_{c}$ is around $44.12 \mathrm{~K}$ for $\mathrm{ErFeO}_{3}$, and around $6.83 \mathrm{~K}$ for $\mathrm{NdFeO}_{3}$.
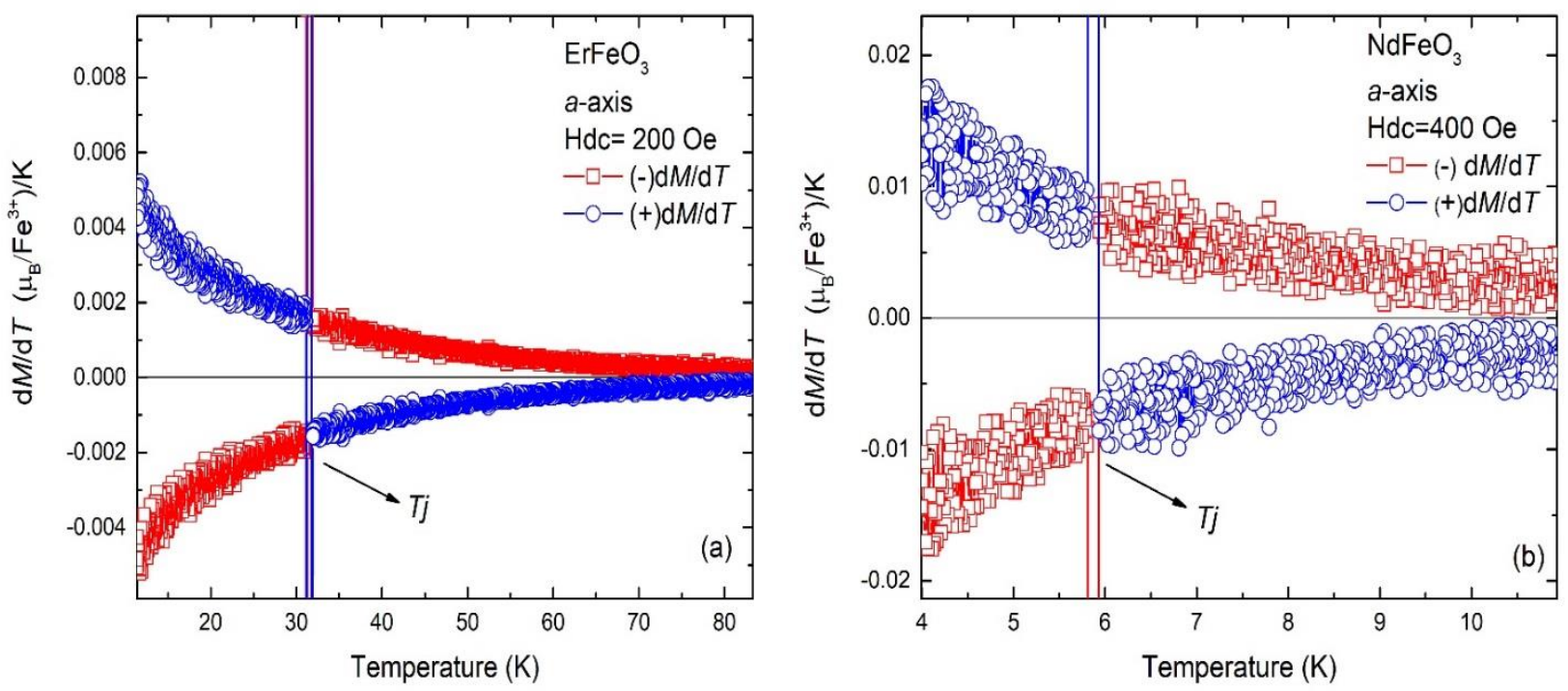

Figure 3. The magnetic moment differentiated vs temperature for both samples, (a) $\mathrm{d} M / \mathrm{d} T$ for $\mathrm{ErFeO}_{3}$ single crystal (b) $\mathrm{d} M / \mathrm{d} T$ for $\mathrm{NdFeO}_{3}$ single crystal.

With further cooling, the $R^{3+}$ moment will become larger than for $\mathrm{Fe}^{3+}$, giving a net magnetic moment opposite to the external field (i.e. negative). The spin-flip occurs when the interaction energy between the net magnetic moment of $R \mathrm{FeO}_{3}$ system and external field overcomes the magnetic anisotropy energy. As a result of that, the magnetic moment of whole sample changes 
into the opposite direction, to be parallel to the external field, at the jump temperature $T j$. Below $T j$, magnetic moment of $\mathrm{Fe}^{3+}$ is in the opposite direction to external field and magnetic moment of $R^{3+}$ is in the direction of external field. Magnetic domains are not expected in the measured fields. The degree of rare earth spin polarization could change as the whole spin system is rotated, as this spin polarization is partly in a metastable state. Indeed, magnetic moment of $R^{3+}$ was shown to change during the more gradual spin reorientation process [14,22]. The metastability is observed through magnetic decay and irreversibility effects in the magnetic moment when cycling the temperature, field or time as shown in Fig. 4.

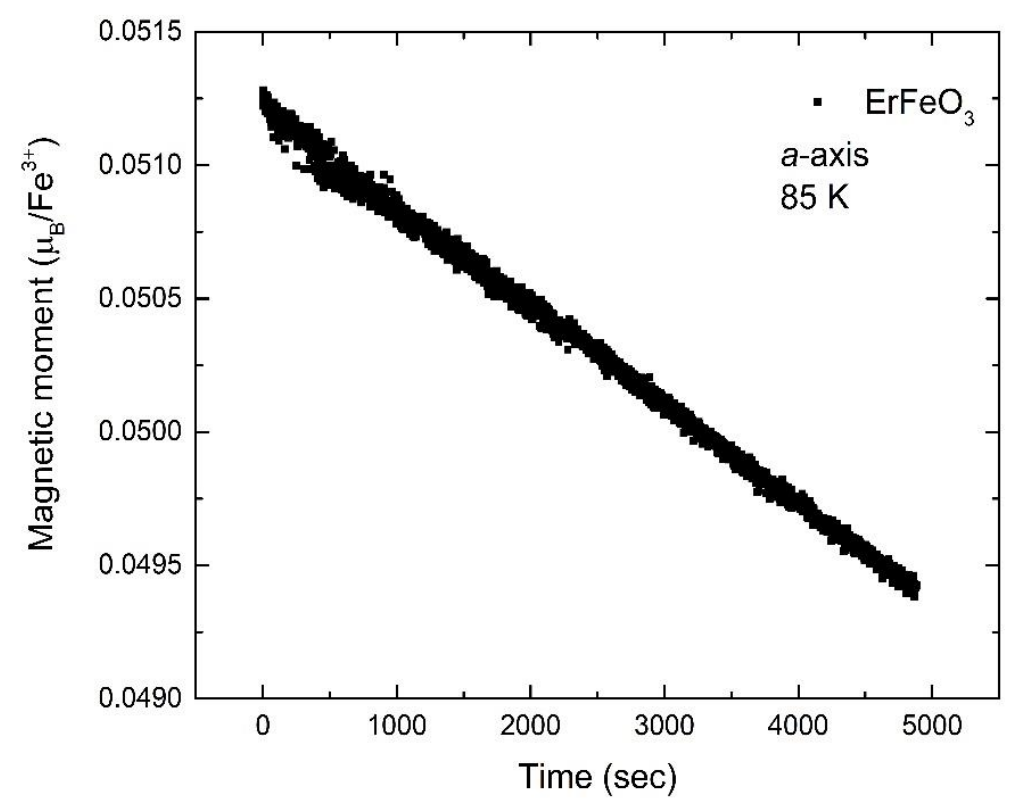

Figure 4. Time-dependence of the magnetic moment for $\mathrm{ErFeO}_{3}$ single crystal at constant temperature and field of $100 \mathrm{Oe}$.

The change in the spin polarization state of $R^{3+}$ is then expected to result in a subtle anomaly in $M$ vs. $T$. Further, the spins could tilt slightly away from the easy axis of anisotropy on the approach to $T j$ from above, because of the increasingly large net magnetic moment of $R^{3+}$ that is in the opposite direction to the field. To see in more detail if there is any magnetic anomaly near $T j, \mathrm{~d} M / \mathrm{d} T$ vs. $T$ was plotted in Fig. 3 (a) and (b) for both $\mathrm{ErFeO}_{3}$ and $\mathrm{NdFeO}_{3}$ respectively. Adding the plot of $-\mathrm{d} M / \mathrm{d} T$ vs. $T$ reveals if $\mathrm{d} M / \mathrm{d} T$ vs. $T$ follows the same form below and above $T j$. Our results in Fig. 3 show no change in $\mathrm{d} M / \mathrm{d} T$ on crossing $T j$, revealing no anomaly expected from the irreversibility effects. 
This result is surprising, as the net magnetic moment of $R^{3+}$ changes in the spin-flip, resulting in the absolute value of its net magnetic moment just above $T j$ to be smaller than just below $T j$ (Fig. 2). Similar happens in the spin reorientation process at higher temperatures [14,22]. However, Fig. 3 shows the temperature dependence of the net magnetic moment is not affected by the spin-flip process. Therefore, the same polarization process of $R^{3+}$ by the ordered $\mathrm{Fe}^{3+}$ occurs above and below $T j$.

\section{Summary}

Our measurements show that the temperature dependence of magnetic moment for both $\mathrm{ErFeO}_{3}$ and $\mathrm{NdFeO}_{3}$ is not affected by the spin-flip process. This is despite the fact that the rare earth spins, polarized by iron, are in a metastable state that results in slight magnetic decay of the total magnetic moment. The absolute value of magnetic moment changes in the spin-flip process, but not its temperature dependence. This indicates the mechanism of polarization of $R^{3+}$ by $\mathrm{Fe}^{3+}$ ions is robust enough to withstand the spin-flip process and the effects that lead to magnetic decay and irreversibilities of the $R^{3+}$ system. 


\section{Reference}

[1] R. L. White, Journal of Applied Physics 40, 1061 (1969).

[2] T. Yamaguchi, Journal of Physics and Chemistry of Solids 35, 479 (1974).

[3] D. Treves, Physical Review 125, 1843 (1962).

[4] H. F. Forestier, H and G. Guiot-Guillain, Comptes Rendus 230, 1844 (1950).

[5] D. Wood, L. Holmes, and J. Remeika, Physical Review 185, 689 (1969).

[6] W. Koehler, E. Wollan, and M. Wilkinson, Physical Review 118, 58 (1960).

[7] G. Gorodetsky, R. Hornreich, I. Yaeger, H. Pinto, G. Shachar, and H. Shaked, Physical Review B 8, 3398 (1973).

[8] G. Gorodetsky, L. M. Levinson, S. Shtrikman, D. Treves, and B. Wanklyn, Physical Review 187, 637 (1969).

[9] K. P. Belov, A. K. Zvezdin, A. M. Kadomtseva, and R. Levitin, Soviet Physics Uspekhi 19, 574 (1976).

[10] E. Holzschuh, A. Denison, W. Kündig, P. Meier, and B. Patterson, Physical Review B 27, 5294 (1983).

[11] V. D. Buchel'nikov, N. Dan'shin, L. Tsymbal, and V. G. Shavrov, Physics-Uspekhi 39, 547 (1996).

[12] Y. B. Bazaliy, L. Tsymbal, G. Kakazei, A. Izotov, and P. Wigen, PhysIical Review B 69, 104429 (2004).

[13] L. T. Tsymbal, Y. B. Bazaliy, V. N. Derkachenko, V. I. Kamenev, G. N. Kakazei, F. J. Palomares, and P. E. Wigen, Journal of Applied Physics 101, 123919, 1 (2007).

[14] S. Yuan, Y. Wang, M. Shao, F. Chang, B. Kang, Y. Isikawa, and S. Cao, Journal of Applied Physics 109, 07E141 (2011).

[15] J. De Jong, A. Kimel, R. Pisarev, A. Kirilyuk, and T. Rasing, Physical Review B 84, 104421 (2011).

[16] B.-G. Park, S. B. Kim, H.-J. Lee, Y. H. Jeong, J.-H. Park, and C.-S. Kim, Journal of Korean Physical Society 53, 758 (2008).

[17] A. Wu, Z. Wang, B. Wang, X. Ban, L. Jiang, J. Xu, S. Yuan, and S. Cao, Solid State Communications 185, 14 (2014).

[18] X. Wang, S. Cao, Y. Wang, S. Yuan, B. Kang, A. Wu, and J. Zhang, Journal of Crystal Growth 362, 216 (2013).

[19] Y. B. Bazaliy, L. Tsymbal, G. Kakazei, and P. Wigen, Journal of Applied Physics 95, 6622 (2004).

[20] H. Shen, Z. Cheng, F. Hong, J. Xu, S. Yuan, S. Cao, and X. Wang, Applied Physics Letters 103, 192404 (2013).

[21] G. Song, J. Jiang, B. Kang, J. Zhang, Z. Cheng, G. Ma, and S. Cao, Solid State Communications 211, 47 (2015).

[22] Y. B. Bazaliy, L. Tsymbal, G. Kakazei, A. Izotov, and P. Wigen, Physical Review B 69, 104429 (2004). 